\title{
Evaluación del uso de la realidad aumentada en la educación musical*
}

EVALUATION OFMUSIC EDUCATION USING AUGMENTED REALITY

AVALIAÇÃO DO USO DE REALIDADE AUMENTADA NO ENSINO DA MÚSICA

\section{Liliana Patricia Amaya Cote** Julian Santiago Santoyo Díaz***}

Cuadernos de Música, Artes Visuales y Artes Escénicas

/ Volumen 12 - Número 1 / Enero - Junio de 2017

/ ISSN 1794-6670/ Bogotá, D.C., Colombia / pp. 143-157

Fecha de recepción: 7 de abril de 2016

Fecha de aceptación: 10 de julio de 2016

Disponible en línea: 20 de febrero de 2017

doi:10.11144/Javeriana.mavae12-1.urae

\footnotetext{
* Artículo de investigación. Este artículo es parte del trabajo de grado en la Maestría de Educación en la Universidad Autónoma de Bucaramanga.

* Maestra en Música, especialista en Necesidades Educativas Especiales y magíster en Educación por la Universidad Autónoma de Bucaramanga (Colombia). Docente de cátedra en la Universidad Industrial de Santander. Correo electrónico: lamaya5@unab.edu.co

*** Ingeniero de Sistemas, especialista en Tecnologías Avanzadas para el Desarrollo de Software por la Universidad Autónoma de Bucaramanga (Colombia), Magíster en Sistemas y Servicios en la Sociedad de la Información por la Universidad de Valencia (España), con especialidad en gestión y desarrollo de servicios y aplicaciones web. Administrador de LMS UNAB virtual y docente cátedra de la Universidad Autónoma de Bucaramanga. Correo electrónico: jsdiaz@unab.edu.co
} 


\section{Resumen}

La presente investigación se centró en el desarrollo de una metodología para usar la técnica de realidad aumentada, con el fin de crear material didáctico musical que permita la promoción de la autonomía en el aprendizaje del estudiante. Partiendo de esta idea, se analizaron y determinaron las herramientas tecnológicas con realidad aumentada que facilitaran la creación del material didáctico multimedia (audio, video, imágenes y modelos 3D) para el aprendizaje y montaje de repertorio musical. A continuación se creó material didáctico con realidad aumentada y se aplicó a un grupo experimental, con el propósito de determinar si existe alguna diferencia entre la implementación de realidad aumentada en la clase de música y el uso del método de enseñanza tradicional. Finalmente, se evaluó el efecto a partir de una rúbrica a los dos grupos: control y experimental, además de un cuestionario de satisfacción para el grupo experimental, que llegó a determinar las contribuciones del material didáctico en la realidad aumentada en el aprendizaje autónomo.

Palabras claves: realidad aumentada; material didáctico; ensamble musical; aprendizaje autónomo.

\section{Abstract}

This research focused on the development of a methodology for using the technique of augmented reality $(A R)$ in order to create musical didactic material that allows the promotion of autonomy in student learning. Starting from this idea, he analyzed and identified with augmented reality technology tools that facilitate the creation of multimedia teaching materials (audio, video, images and 3D models) for learning and assembling musical repertoire. Then teaching materials created with augmented reality, applying this material to an experimental group in order to determine whether there is any difference between the use of augmented reality in music class and the use of traditional teaching method. Finally, the impact from one heading to the two groups were evaluated: control and experimental plus a satisfaction questionnaire to the experimental group, which came to determine the contributions of the teaching material in augmented reality on autonomous learning.

Keywords: augmented reality; teaching materials; musical ensemble; independent learning.

\section{Resumo}

Esta pesquisa focada no desenvolvimento de uma metodologia para usar a técnica de realidade aumentada (AR), a fim de criar material didático musical que permite a promoção da autonomia na aprendizagem dos alunos. A partir desta idéia, ele analisados e identificados com ferramentas aumentada tecnologia de realidade que facilitam a criação de materiais multimédia de ensino (áudio, vídeo, imagens e modelos 3D) para a aprendizagem e montagem de repertório musical. Em seguida, ensinando materiais criados com realidade aumentada, aplicando-se este material a um grupo experimental, a fim de determinar se existe alguma diferença entre o uso de realidade aumentada na classe de música eo uso de método de ensino tradicional. Finalmente, foram avaliados o impacto de um título para os dois grupos: controle e experimental, mais um questionário de satisfação para o grupo experimental, que veio para determinar as contribuições do material de ensino em realidade aumentada na aprendizagem autônoma.

Palavras chave: realidade aumentada; materiais pedagógicos; conjunto musical; aprendizagem autônoma. 


\section{INTRODUCCIÓN}

La formación musical se hace primordial en los primeros años de vida, no solo porque motiva, incentiva la disciplina hacia su instrumento y ayuda a la socialización al tocar en grupo, sino porque permite la evolución cerebral. Según Collins (2014), al tocar un instrumento se involucran todas las partes del cerebro, en especial el córtex visual, auditivo y motor, además aumenta el volumen y la actividad del cuerpo calloso que facilita resolución de problemas, rápida y creativamente. Esto muestra cómo los músicos son mejores creando, registrando y recuperando recuerdos, dado que tienen una gran capacidad para la memoria, lo cual permite fortalecer el cerebro en todas sus funciones y ayuda a desarrollar otras áreas del conocimiento.

En Colombia, se debe propender a una educación musical constante y rigurosa, donde se logre enriquecer los procesos cognitivos, actitudinales y procedimentales de los estudiantes, fomentar disciplina, formar a partir del arte ciudadanos universales, sensibles y mejores seres humanos con su entorno. Pero la solución no la tiene el Ministerio de Educación Nacional, ni las instituciones, sino que la posibilidad de que esto mejore está también en los maestros, por lo que de ellos se requiere que 1) muestren a la comunidad educativa el trabajo del aula, 2) fomenten clases de instrumento participativas, autónomas y motivantes para los educandos y

3) despierten el interés de los estudiantes a partir de la utilización de recursos y herramientas digitales TIC (tecnologías de la información y de la comunicación) (Ronderos y Mantilla 1997).

La realidad aumentada es una tendencia en ascenso, innovadora y motivadora, ya que logra integrar lo real con lo virtual en un solo lugar (Navarro 2014). ¿A qué ser humano no le gusta "jugar" con lo virtual? Por eso cada día nuestros jóvenes pasan horas en el computador, tableta o celular con su juego favorito. La realidad aumentada posee una capacidad de adaptación a cualquier espacio cotidiano y promete acercarse más a lo inimaginable y desconocido de nuestro entorno. Según el mismo autor, el futuro está en la realidad aumentada, porque permite una vivencia real e inmediata con manos libres. Si esta es la tendencia y se logra llevar a las aulas de clase, resultará ser un elemento importante de innovación educativa que tendrá resultados significativos a largo plazo en el aprendizaje de los estudiantes, facilitará las prácticas instrumentales y mejorará las clases.

Lastimosamente, el desconocimiento por parte de los profesores sobre los materiales y recursos tecnológicos que pueden hacer de una clase de música una clase atractiva, interesante y divertida para nuestros estudiantes es un factor importante en la subutilización de las tecnologías en la enseñanza musical (Peula et al. 2006). Los maestros deben olvidar un poco el marcador y el tablero e ir más allá, llevar a los estudiantes a la experimentación, a que conozcan a través de sus cinco sentidos el mundo y así aprender de forma autónoma.

En este contexto, surgió este proyecto Material didáctico en realidad aumentada como apoyo a la educación musical en jóvenes de octavo grado, que es de interés por su propuesta innovadora en el uso de las nuevas tecnologías emergentes y sus alcances en el aula de clase frente al método tradicional, que, aunque utiliza en ocasiones las TIC (editores de partitura y material multimedial), no emplea material con el que los estudiantes puedan interactuar con la música. Y por otro lado, y no menos importante, enriquecer las prácticas docentes a partir de la realidad aumentada en el aula, que sirven para la música o para otras disciplinas dentro de la misma institución. De las premisas anteriores nació el planteamiento de la pregunta de investigación que orienta este proyecto: ¿existe una diferencia significativa en la capacidad de ensamble rítmico melódico cuando se utiliza un material didáctico basado en realidad 
aumentada o el método tradicional de enseñanza en jóvenes de octavo grado? La pregunta de investigación planteada buscó la relación entre las siguientes dos variables: 1) capacidad de ensamble rítmico melódico y 2) material basado en realidad aumentada para lograr aprendizaje autónomo.

\section{MÉTODO}

Se planteó desarrollar una investigación cuantitativa, cuasiexperimental por la no aleatorización de los sujetos de cada grupo (Hernández 1998). Estos se seleccionaron desde antes de la prueba piloto por parte de la institución y el grupo A fue el grupo control y el grupo B fue el grupo experimental. El enfoque cuantitativo se escogió porque permite probar si la variable independiente causa efecto en la dependiente, y para esto fue necesario la recolección de datos antes y después del tratamiento, con el fin de probar la hipótesis que es la siguiente: el uso de la realidad aumentada como herramienta tecnológica, pedagógica y motivacional en el aula de música permite asegurar el aprendizaje autónomo en el montaje de repertorio musical en los jóvenes de octavo grado.

\section{POBLACIÓN}

El tipo de muestreo es igual a la población del proyecto, y en total se contó con la participación activa de 56 estudiantes, de los cuales 32 son hombres y 24 mujeres, con edades entre los 13 y 15 años. Todos los estudiantes son del grado octavo de una institución privada de Bucaramanga.

\section{INSTRUMENTO DE RECOLECCIÓN DE DATOS}

La evaluación en la música debe generar un "cambio" en el proceso de apropiación de un instrumento o voz dentro de un ensamble vocal o instrumental (Duke 2010), por tanto, es importante utilizar instrumentos que permitan no solo valorar eficientemente una canción, sino también identificar qué nuevos aprendizajes o técnicas adquirió un estudiante en ese desarrollo. A continuación, los dos instrumentos que se utilizaron en el proyecto: rúbrica de evaluación musical y cuestionario.

\section{RÚBRICA}

Teniendo en cuenta lo dicho por Saunders y Holahan (1997), para tener un excelente instrumento de evaluación musical, la rúbrica debe contener criterios específicos sobre la capacidad de ejecución de un instrumentista: “Los jurados, entonces, describen lo que escucharon, sin indicar si les gustó o no, o si están de acuerdo o en desacuerdo (261). Según Colwell (2002), para que una rúbrica sea efectiva, debe ser una lista de chequeo, que, además de que arroja resultados con validez y confiabilidad, sirven de retroalimentación para cada estudiante. A causa de lo anterior, se escogió una rúbrica aplicada a estudiantes de las bandas de Carolina del Sur (South Carolina Band Directors Association 2015), y se ubica la rúbrica para vientos y para percusión. Para este proyecto, se tradujeron los criterios al español y se dejaron intactos la escala de valoración y el puntaje final. Los aspectos que se evaluaron son estos: técnica, precisión rítmica, 
tono, interpretación, entonación, y otros factores, como expresión musical y seguridad. Esta rúbrica en el pretest se aplicó por la docente de música titular y en el postest se invitaron jurados externos para valorar a los dos grupos, con el fin de responder a la pregunta problema de este proyecto, lo anterior teniendo en cuenta lo que dice Bergee (2003) sobre tener un instrumento de evaluación que diera validez y confiabilidad: 1) cantidad de jurados, 2) forma de la evaluación (escala de valoración y sugerencias) y 3) experiencia del jurado en cada instrumento. En esta rúbrica, se dio un valor de 1 a 5 en cada uno de los criterios, donde 5 es la calificación más alta y 1 la más baja, para un total de 30 puntos (tablas 1 y 2 ).

TABLA 1. DISCRIMINACIÓN RÚBRICA POR CRITERIOS

\begin{tabular}{ll} 
Técnica & Notas correctas \\
& Articulación \\
& Dedos y posición del instrumento \\
\hline Precisión rítmica & Valor nota \\
& Valor silencio \\
& Pulso \\
\hline Tono & Respiración \\
& Embocadura \\
& Digitación \\
& Postura instrumento para una mejor ejecución \\
\hline Interpretación & Dinámicas \\
& Balance \\
& Fraseo \\
& Estilo \\
& Tempo \\
\hline Entonación & Entonación individual \\
& Entonación con acompañamiento \\
\hline Otros factores & Seguridad \\
Apariencia \\
Expresión musical
\end{tabular}

TABLA 2. RANGO DE CALIFICACIÓN DE LA RÚBRICA

\begin{tabular}{ll} 
Rango & Puntuación \\
\hline Superior & $27-30$ puntos \\
Excelente & $21-26$ puntos \\
Bueno & $15-20$ puntos \\
Básico & $9-14$ puntos \\
Bajo & $0-8$ puntos
\end{tabular}




\section{CUESTIONARIO}

Con este instrumento, se analizó la percepción del estudiante en torno a la realidad aumentada, además de identificar su forma de aprender y estudiar la música. El cuestionario fue anónimo y no evaluable. Las preguntas se distribuyeron de la siguiente forma: 1) pregunta de selección múltiple, 2) una pregunta dicotómica, 3) nueve preguntas con escalas de valoración tipo Likert y 4) una pregunta abierta de sugerencias. Se entregó el enlace realizado en Google Forms $₫$ solo para los estudiantes del grupo experimental.

\section{PROCEDIMIENTO}

\section{CREACIÓN DE MATERIAL MUSICAL}

En primer lugar, se buscó un tema musical que cumpliera con los lineamientos y tópicos propuestos en el plan de asignatura del grado octavo, para ello, y como el objetivo del periodo era reconocer el jazz como influencia musical de géneros actuales, se decidió arreglar el tema Happy (Williams 2013) para ensamble de cinco flautas dulces, una guitarra, piano y percusión corporal.

\section{CREACIÓN DEL MATERIAL DIDÁCTICO}

Luego de definir la aplicación que facilitó el material didáctico con realidad aumentada, se procedió a crear los marcadores con realidad aumentada, para lo cual se debía tener a la mano todo el material multimedia, que iba a ser parte del material dado a los estudiantes. Este consistía en videos lúdicos sobre cada línea melódica por ejecutar en el instrumento, audios de la partitura y partitura en general. Con estos recursos, se pretendió armar marcadores eficientes que sirvieron para el aprendizaje de canciones y el estudio particular en casa, por esto se escogió la aplicación ARcrowd (Verona y Pino 2015), que permite colocar los marcadores con realidad aumentada dentro de un solo ARbook, los cuales se pueden empotrar en otras páginas web. Para que los estudiantes visualizaran los marcadores, se creó una página en Wix (http://lilianamayamusica.wix. com/ramusica), donde se ordenaron los visores por partes de la canción y el instrumento.

\section{APLICACIÓN DEL MATERIAL DIDÁCTICO}

Como se describió, se utilizó el material didáctico con el propósito de que ellos conocieran la realidad aumentada a partir de juegos, ejercicios de instrumentos musicales virtuales (piano) y conocimientos teóricos, que sirvieron de base para el posterior montaje de la canción del periodo (figura 1).

Para que la proyección del marcador en el visor fuera de la mejor calidad, se pegaron los marcadores en un cartón, con el propósito de que estos tuvieran estabilidad. Igualmente, se les sugirió a los estudiantes pegar el marcador con cinta en una pared que le diera luz, con el fin de que la cámara web de los classmate (computadores portátiles) pudiera capturar la imagen con claridad y le diera estabilidad a la imagen (figuras 2 y 3 ). 


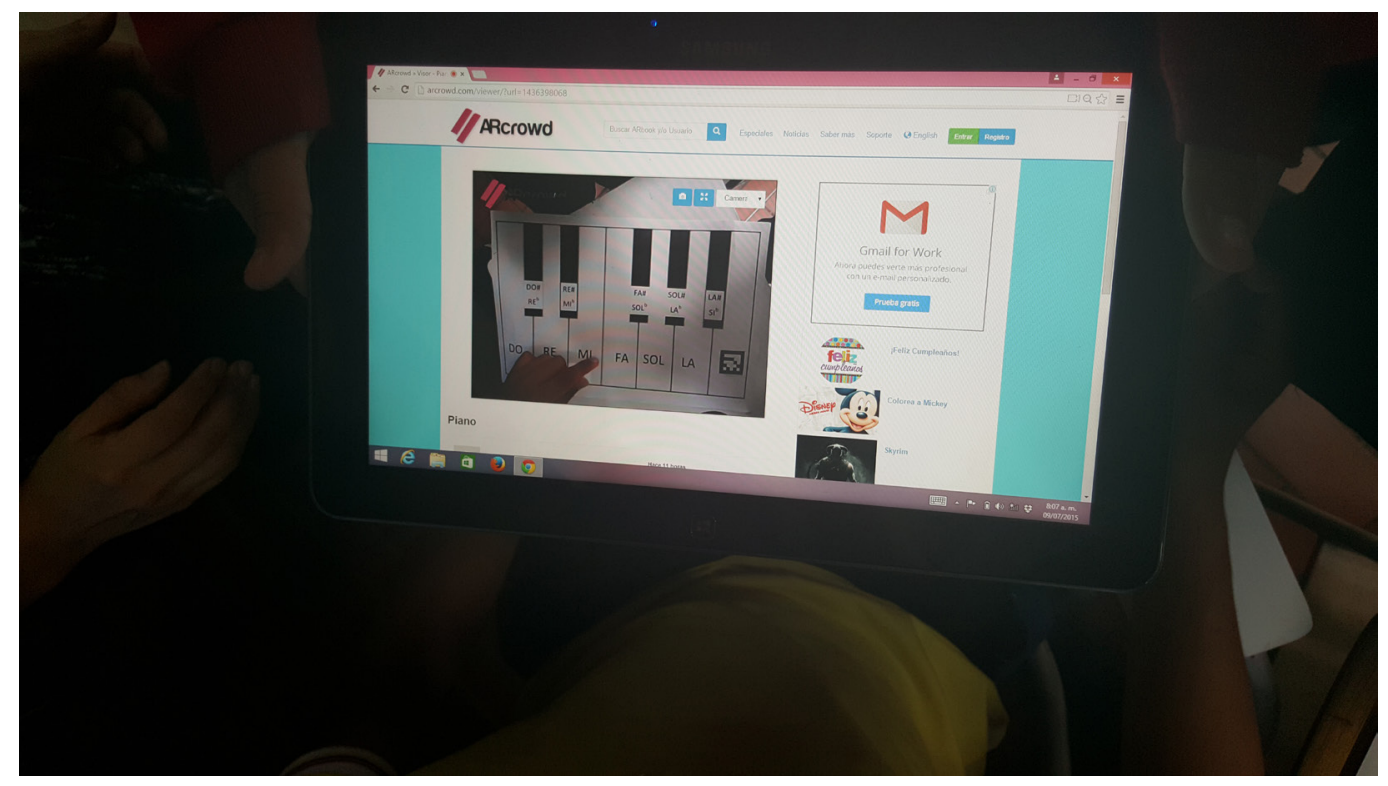

Figura 1. Los estudiantes experimentan con un piano realizado a partir de doce marcadores de realidad aumentada. Al colocar los marcadores, estos lograban la producción de sonido.

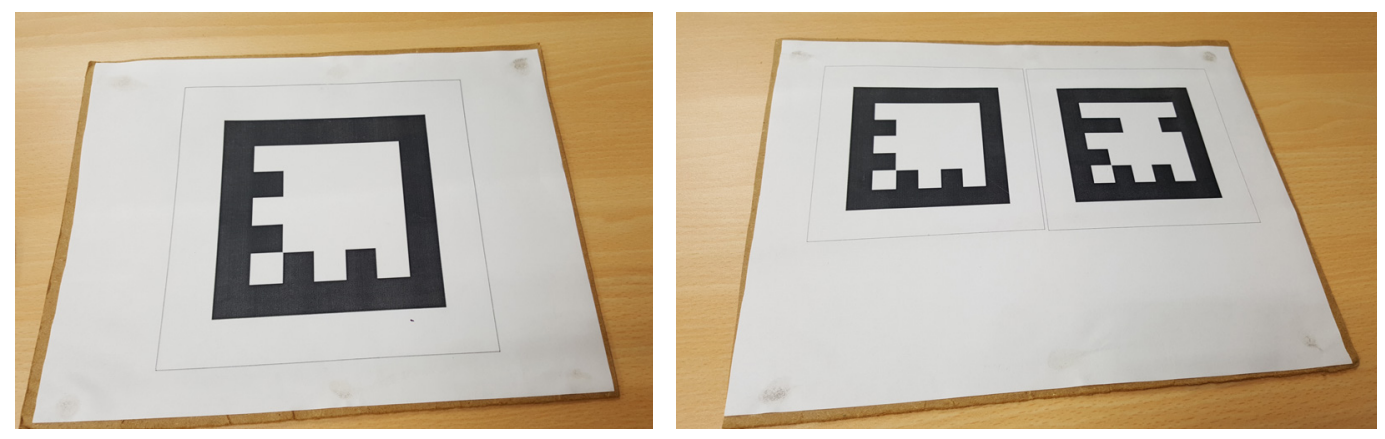

Figura 2. Marcadores que se entregaban a los estudiantes. El marcador de la izquierda se utilizaba para visualizar partituras o videos y en el marcador de la derecha se podía ver la partitura más pequeña y el audio a la vez.

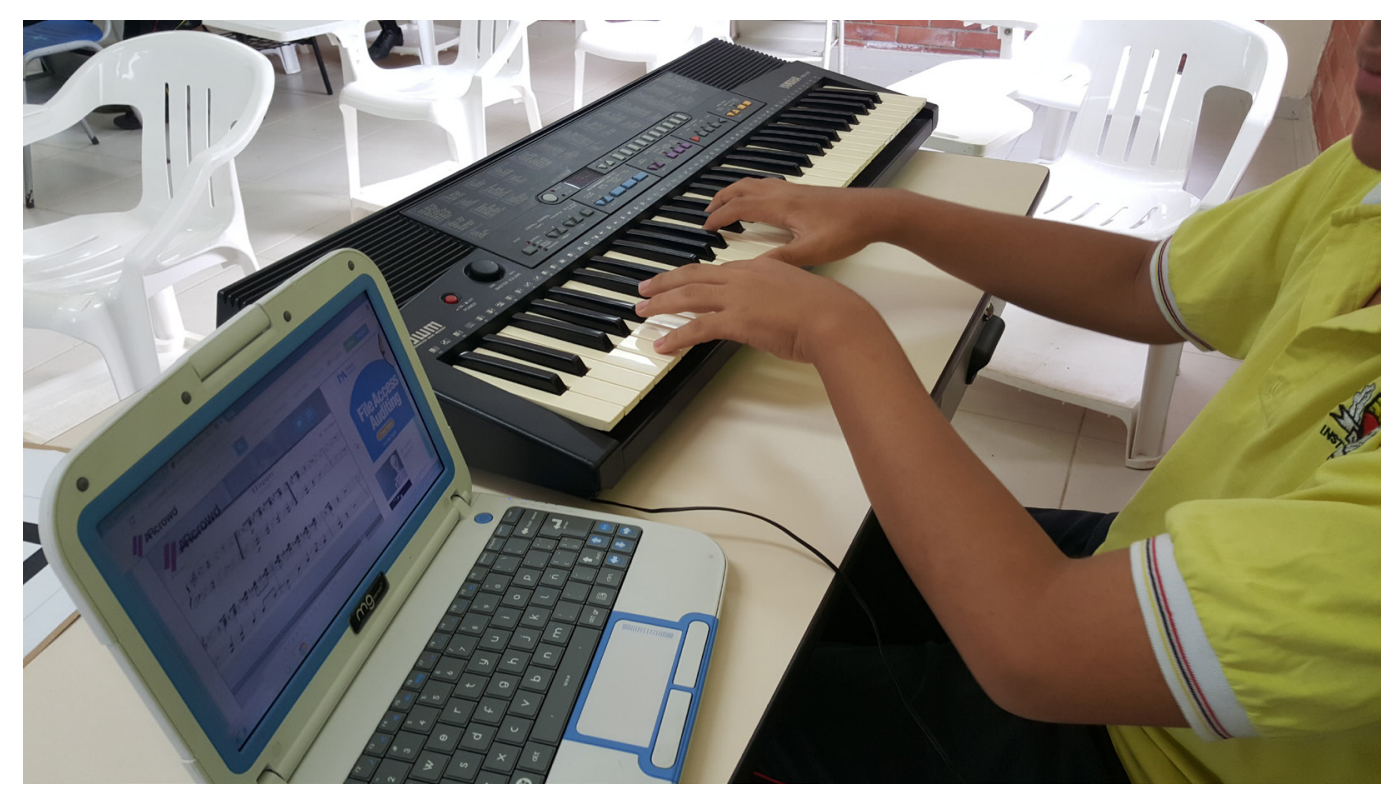

Figura 3. Estudiante de piano estudiando su partitura con realidad aumentada. Si le da vuelta al marcador, podrá ver la partitura y escuchar a la vez el audio. 


\section{RESULTADOS}

A partir de los resultados de las dos mediciones (pretest y postest), se realizó un análisis utilizando la prueba prueba t-Student para muestras independientes y muestras relacionadas con la ayuda del software SPSS.

\section{ANÁLISIS DE MUESTRAS INDEPENDIENTES PRETEST}

Con el fin de verificar la homogeneidad de ambos grupos, se aplicó el análisis de muestras independientes. A continuación, el histograma que muestra el comportamiento normalizado de los datos (figura 4).

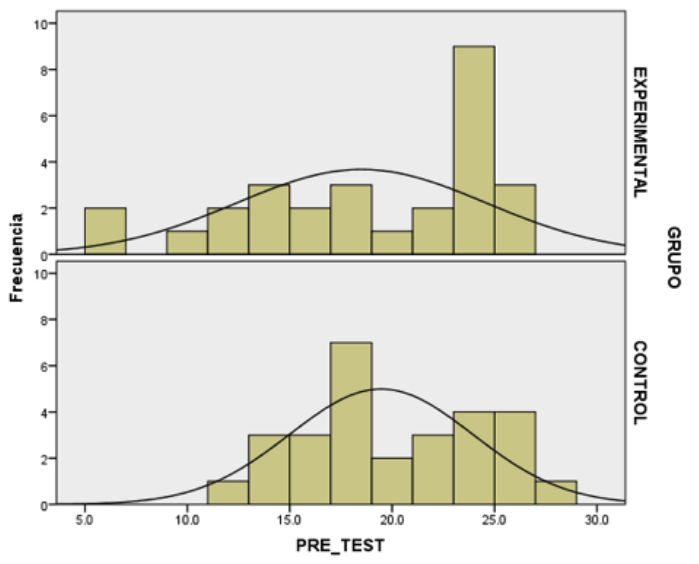

Figura 4. Histograma de los resultados pretest.

El resultado de la prueba t-Student es 0.502 .

p-valor $=0.502>\nabla=0.05$

Esto concluye que, al no existir una diferencia significativa entre las calificaciones del grupo control y el grupo experimental en el pretest, los dos grupos son homogéneos.

\section{ANÁLISIS DE MUESTRAS INDEPENDIENTES POSTEST}

Para este análisis, se tomaron los resultados arrojados en la última medida, es decir, el postest, la prueba después del tratamiento. Para conocer si hubo una diferencia significativa en el aprendizaje autónomo de un ensamble musical, se ha trabajado el grupo control con el método tradicional y el grupo experimental con el material didáctico con realidad aumentada. En todos los análisis, se pretendió descartar la hipótesis nula que es esta: no existe una diferencia significativa en la capacidad de ensamble rítmico melódico cuando se utiliza un material didáctico basado en realidad aumentada y el método tradicional de enseñanza en jóvenes de octavo grado o dar viabilidad a la principal hipótesis ya presentada anteriormente. Para esto, es importante tener en cuenta el nivel alfa o el nivel de significancia en los resultados, que será de $5 \%$ (0.05). 
El resultado del nivel de significancia es 0.038 .

p-valor $=0.038 \quad<\otimes=0.05$

Esto concluye que existe una diferencia significativa entre las calificaciones del grupo control y el grupo experimental.

\section{ANÁLISIS DE LA PRUEBA T-STUDENT DE MUESTRAS RELACIONADAS}

Esta prueba se utiliza para estudios de tipo longitudinal, es decir, para medidas en dos momentos temporales distintos de pretest y postest.

\section{ANÁLISIS DE LA PRUEBA T-STUDENT DE DOS MUESTRAS RELACIONADAS EN EL GRUPO CONTROL}

Se realizó la prueba t-Student para dos muestras relacionadas. Los resultados muestran que la media en el pretest es de 19.46 y en el postest es de 15.79, es decir, la media disminuye en el grupo control; sin embargo, hay que revisar si el nivel es significativo. A continuación, en la figura 5, se pueden ver los resultados.

\begin{tabular}{|c|c|c|c|c|c|c|c|c|}
\hline \multicolumn{9}{|c|}{ Prueba de muestras relacionadas } \\
\hline & \multicolumn{5}{|c|}{ Diferencias relacionadas } & \multirow[b]{3}{*}{$\mathrm{t}$} & \multirow[b]{3}{*}{$\mathrm{gl}$} & \multirow{3}{*}{$\begin{array}{l}\text { Sig. } \\
\text { (bilateral) }\end{array}$} \\
\hline & \multirow[b]{2}{*}{ Media } & \multirow{2}{*}{$\begin{array}{l}\text { Desviación } \\
\text { tip }\end{array}$} & \multirow{2}{*}{$\begin{array}{l}\text { Error típ. de } \\
\text { la media }\end{array}$} & \multicolumn{2}{|c|}{$\begin{array}{l}95 \% \text { Intervalo de confianza } \\
\text { para la diferencia }\end{array}$} & & & \\
\hline & & & & Inferior & Superior & & & \\
\hline $\begin{array}{c}\text { Par } 1 \\
\text { PRE - POS }\end{array}$ & 3.679 & 5.605 & 1.059 & 1.505 & 5.852 & 3.473 & 27 & .002 \\
\hline
\end{tabular}

Figura 5. Prueba t-Student para dos muestras relacionadas en el grupo control.

La significancia es el 0.002, es decir, la hipótesis nula se rechaza y se acepta la hipótesis alterna que dice que en el grupo control hay una diferencia significativa en el uso del método tradicional entre sus dos mediciones; sin embargo, la media disminuye significativamente en este grupo.

\section{ANÁLISIS PRUEBA T-STUDENT DE DOS MUESTRAS RELACIONADAS EN EL GRUPO EXPERIMENTAL}

Los resultados muestran la media en el pretest que es de 18.5 y en el postest que es de 19.82, es decir, la media aumenta; sin embargo, hay que revisar si el nivel es significativo. A continuación, en la figura 6 , se pueden visualizar los resultados.

\begin{tabular}{|c|c|c|c|c|c|c|c|c|}
\hline \multicolumn{9}{|c|}{ Prueba de muestras relacionadas } \\
\hline & \multicolumn{5}{|c|}{ Diferencias relacionadas } & \multirow[b]{3}{*}{$\mathrm{t}$} & \multirow[b]{3}{*}{ gl } & \multirow{3}{*}{$\begin{array}{c}\text { Sig. } \\
\text { (bilateral) }\end{array}$} \\
\hline & \multirow[b]{2}{*}{ Media } & \multirow{2}{*}{$\begin{array}{l}\text { Desviación } \\
\text { tip }\end{array}$} & \multirow{2}{*}{$\begin{array}{l}\text { Error típ. de } \\
\text { la media }\end{array}$} & \multicolumn{2}{|c|}{$\begin{array}{l}95 \% \text { Intervalo de confianza } \\
\text { para la diferencia }\end{array}$} & & & \\
\hline & & & & Inferior & Superior & & & \\
\hline $\begin{array}{c}\text { Par } 1 \\
\text { PRE - POS }\end{array}$ & -3.000 & 7.547 & 1.426 & -5.927 & -.073 & -2.103 & 27 & .045 \\
\hline
\end{tabular}

Figura 6. Prueba t-Student para dos muestras relacionadas en el grupo experimental. 
La significancia es de 0.045 , es decir, la hipótesis nula se rechaza y se concluye que existe una diferencia significativa en las mediciones pretest y postest en el grupo experimental y los estudiantes en efecto sí subieron la media.

\section{PRESENTACIÓN DE LOS RESULTADOS DEL CUESTIONARIO}

La recolección de la información se llevó a cabo el 5 de octubre de 2015 después de finalizado el tercer periodo, con los 28 estudiantes del grupo experimental a los que se les aplicó el tratamiento. La primera sección de preguntas indagó sobre el uso y la frecuencia de la tecnología, específicamente de internet. A continuación, el cuestionario de satisfacción giró en torno a la aplicación de la realidad aumentada en la clase de música. La pregunta n. ${ }^{\circ} 4$ cuestiona sobre el uso de la realidad aumentada, solo 22 \% de la población le da una valoración de 4 y 5 . Sin embargo, a pesar de ser similar la pregunta.$^{\circ} 4$ a la n. ${ }^{\circ} 11$, los estudiantes sí le dan un valor alto al uso de material didáctico con realidad aumentada en el aprendizaje de la música, con $44 \%$ en el valor 4 .

En cuanto a las herramientas dadas para el uso de la realidad aumentada, se preguntó en tres ocasiones si estas fueron las necesarias. La pregunta n. ${ }^{\circ} 7$ indaga sobre la aplicación ARcrowd utilizada para esta función, y los resultados rondan entre el valor 2 y el 4 sobre 5 .

Con el fin de saber si la información que se suministró era la correcta para el uso de la realidad aumentada, se les preguntó en el cuestionario y se evidenció que 57 \% fue favorable en el valor 4 de 1 a 5 .

Otro elemento importante para el uso correcto de la realidad aumentada era la página web que se creó con el fin de que existiera un acceso desde cualquier lugar y donde podían los estudiantes encontrar todos los visores y marcadores. Los resultados se mantienen entre los valores 3 y 4 , con $39 \%$ cada uno.

Un componente muy importante en el uso de la realidad aumentada es el incremento de la motivación, que despierta a la vez un mejor aprendizaje (las siguientes dos preguntas se enfocan en eso). La pregunta n. ${ }^{\circ} 6$ indaga sobre la comodidad en el uso de la realidad aumentada. El $43 \%$ de los estudiantes le dio un valor de 3 sobre 5, y los otros valores están repartidos equitativamente.

Al finalizar el cuestionario, se dejó una pregunta abierta con el fin de que los estudiantes pudieran sugerir y comentar sobre su experiencia en el uso de material didáctico con realidad aumentada en la clase de música, lo cual puede dar luces sobre la efectividad de la herramienta y su posible uso futuro. A continuación, los comentarios de 10 de los 28 estudiantes (tabla 3 ). 
TABLA 3. COMENTARIOS REALIZADOS AL FINAL DEL CUESTIONARIO DE SATISFACCIÓN

Ninguno, me parece que así está bien la herramienta.

Se necesita más tiempo en clase para trabajar con la herramienta.

Es fácil de manejar.

Que sea una forma más fácil de utilizar.

Sugiero que el visor fuera más rápido.

Buscar una manera más fácil para sostener los marcadores y que se mejore la lectura del computador, ya que a veces no lo leía bien y era complicado visualizar la partitura.

No tengo sugerencias sobre el uso de la realidad aumentada, me parece que es un buen medio de aprendizaje.

Que todos tengan un computador para el uso de la realidad aumentada.

Tal cual está bien, no creo que necesite sugerencias.

Que la aplicación ARcrowd fuera un poco más rápida.

\section{DISCUSIÓN}

En general, a partir de esta investigación, se pudo conocer los alcances de la realidad aumentada como tecnología emergente. Se reconoce, por tanto, que con el auge de la tecnología que dará nuevos recursos para el aula los docentes de música podrán utilizar más fácilmente esta y otras tecnologías.

\section{APLICACIÓN EFECTIVA PARA EL USO DE REALIDAD AUMENTADA EN LA MÚSICA}

Como se argumentó en el apartado de limitaciones y delimitaciones, existen algunas aplicaciones que pueden servir para realizar material didáctico con realidad aumentada; sin embargo, todas estas aplicaciones no cuentan con la fácil creación del material o no se pueden generar libros ARbook completos para que los estudiantes puedan acceder a sus visores. Entre todas las aplicaciones que se probaron, la aplicación que se ajustaba a los anteriores requerimientos, tales como fácil creación de marcadores, generador de visores, creación de ARbook y soporte a diferentes formatos de archivo (imagen, sonido, video y modelos 3D), era la plataforma ARcrowd. Entre otras funciones, la plataforma contaba con estadísticas de sus visores, lo cual permitió conocer con qué frecuencia ingresaban los estudiantes y qué visores estudiaban desde sus casas.

\section{DISEÑO DE MATERIAL DIDÁCTICO}

Para el proyecto se diseñó material con marcadores en realidad aumentada, con el propósito de abarcar cuatro etapas en el desarrollo del aprendizaje autónomo teniendo en cuenta el método Orff para el montaje de ensambles musicales: 1) material didáctico para la correcta utilización de la realidad aumentada, 2) material didáctico con nociones musicales básicas para la interpretación de un ensamble, 3) material didáctico para el aprendizaje autónomo de la canción por partes y 4) material didáctico con el ensamble final. 
Se realizó como resultado de una propuesta didáctica un folleto que se entregó al grupo experimental como parte de una "campaña" sobre la plataforma ARcrowd y de la buena utilización de la realidad aumentada. Para este material, se crearon ocho marcadores en un solo ARbook con su propio visor de realidad aumentada (http://arcrowd. com/viewer/?url=1421680319).

\section{MATERIAL DIDÁCTICO CON NOCIONES MUSICALES BÁSICAS PARA LA INTERPRETACIÓN DE UN ENSAMBLE}

En las siguientes sesiones del proyecto, para el grupo experimental, se crearon marcadores con las notas musicales, las figuras y los silencios, escalas en el instrumento, partituras de canciones con realidad aumentada. Se realizó un piano con realidad aumentada que emitía sonidos de las notas a partir de marcadores superpuestos en la hoja donde estaba un piano dibujado (figura 7) (http://arcrowd.com/viewer/?url=1436398068).

\section{MATERIAL DIDÁCTICO PARA EL APRENDIZAJE AUTÓNOMO DE LA CANCIÓN POR PARTES}

A partir del ensamble musical, se crearon marcadores para el aprendizaje de la canción por partes de dos formas: 1) formato video: se grabaron videos que se incluyeron en cada marcador con el fin de ser ejemplo en la interpretación de la obra musical y 2) formato imagen y audio: el editor de partituras arrojaba el archivo en MP3, el cual se utilizaba junto con la partitura para que el estudiante al colocar los dos marcadores juntos lograra escuchar y observar la partitura.

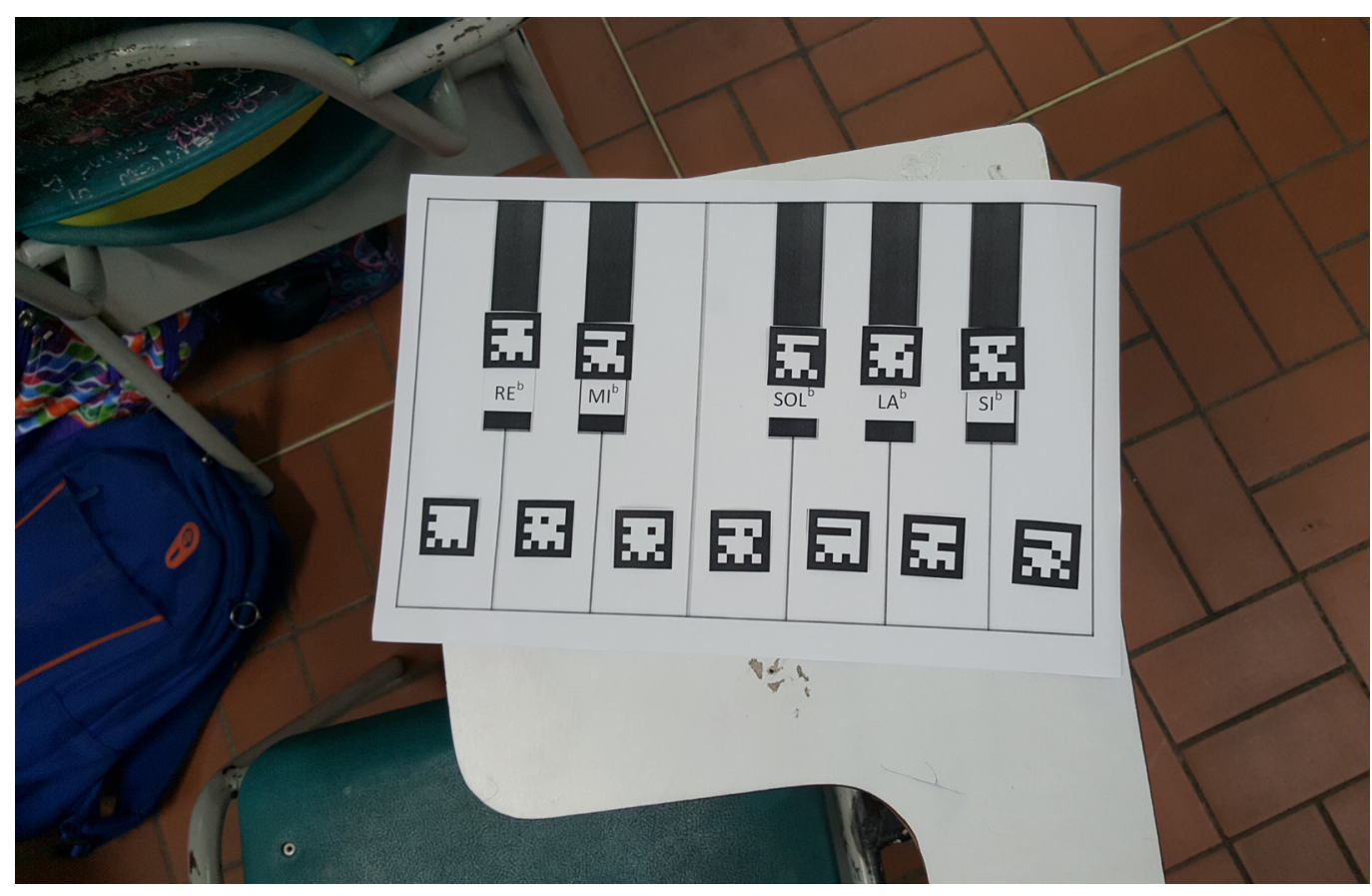

Figura 7. Piano con realidad aumentada. 


\section{MATERIAL DIDÁCTICO CON EL ENSAMBLE FINAL}

Se creó el material didáctico con características lúdicas donde los estudiantes a partir de los visores podían interactuar colocando a sonar todos los instrumentos del ensamble. Igualmente podían escuchar la partitura sin su instrumento para practicar y así mejorar en sus capacidades de ensamble.

\section{APLICACIÓN DEL MATERIAL DIDÁCTICO}

A partir del diseño del material didáctico con realidad aumentada y teniendo en cuenta lo programado en la metodología para la aplicación del tratamiento según el modelo pedagógico de la institución, se llevó a cabo el aprendizaje autónomo con la realidad aumentada en dos etapas: 1) enseñanza de la correcta utilización de la realidad aumentada y nociones básicas y 2) aprendizaje del ensamble musical Happy. Posteriormente, se realizaban revisiones por estudiante y en ensamble en las fases de evaluación y apropiación.

\section{LA REALIDAD AUMENTADA Y LA ENSEÑANZA DE NOCIONES BÁSICAS DE LA MÚSICA}

El primer día de clase del periodo se entregaron siete marcadores impresos a cada estudiante para utilizarlos en el folleto realizado con el fin de que tuvieran un primer acercamiento a la realidad aumentada. Esto despertó gran curiosidad entre los estudiantes, puesto que a través de marcadores podían ver videos, imágenes en $3 \mathrm{D}$ y audio. A partir de ahí, se utilizaron las siguientes dos semanas para preparar al estudiante en el uso del ensamble que se iba a interpretar en el periodo con ayuda de la realidad aumentada.

\section{APRENDIZAJE DEL ENSAMBLE MUSICAL HAPPY}

Después de finalizar esta fase, se dividieron los estudiantes por cuerdas: flauta 1 a la 5, guitarra, piano y percusión de acuerdo con sus habilidades y gustos musicales. Se dedicó tiempo al montaje del primer ensamble por cuerdas de instrumentos utilizando 8 classmate. Se le entregó a cada líder de cuerda los marcadores grandes para que entre ellos escucharan, vieran y leyeran la partitura.

Después se realizó la evaluación de la meta, es decir, se evaluó según el modelo pedagógico el ensamble de la canción, posteriormente se llevó a cabo el repaso del ensamble para que los estudiantes mejoraran en la ejecución del ensamble. Por último, se desarrolló la apropiación según el modelo pedagógico con el fin de cerrar ese día el tercer periodo académico.

\section{EVALUACIÓN DE LA CONTRIBUCIÓN A LA REALIDAD AUMENTADA EN LA MÚSICA}

Después de finalizado el tercer periodo académico, se tomó una última medida para evaluar si existía una diferencia significativa en el uso de la realidad aumentada con el método tradicional. Como ya se explicó, los estudiantes fueron evaluados por pares externos. 
En el análisis de las muestras independientes, se observó que el pretest determinó que los grupos eran homogéneos y que existía una diferencia significativa en el uso de la realidad aumentada frente al método tradicional con los resultados del postest, puesto que los estudiantes del grupo experimental mejoraron frente al grupo control.

Por otra parte, en el análisis de las muestras relacionadas, se observó que, aunque en el grupo control hubo una diferencia significativa entre las calificaciones del pretest y postest, la media no subió sino que por el contrario disminuyó, lo cual quiere decir que los estudiantes desmejoraron en su desempeño musical solo con la utilización del método tradicional para aprender un ensamble musical. Lo contrario pasó en el grupo experimental que alcanzó una diferencia significativa, sí aumentó la media y la mayoría de sus estudiantes mejoraron su desempeño académico. Por tanto, se puede afirmar que la realidad aumentada desarrolla el aprendizaje autónomo en cada estudiante, donde este asume una postura activa de su proceso, manejando los tiempos y objetivos y convirtiendo al docente en un mediador del conocimiento y los recursos.

\section{CONCLUSIONES}

El papel que desempeña la tecnología en nuestra sociedad actualmente es de carácter motivacional. Llegará el momento donde nuestro cuerpo sea parte de ella y ella parte de nosotros. Por eso la educación debe buscar y probar nuevas formas para llegar con la tecnología al conocimiento a partir de la motivación que se genera. Se concluye finalmente que este proyecto no solo probó que la realidad aumentada sí es una herramienta de apoyo en la clase de música, sino que es relevante en cuanto al aumento de la motivación en el aprendizaje de piezas musicales y propicia la colaboración, la cooperación y la convivencia al trabajar en grupo para lograr un aprendizaje. Igualmente, es relevante en cuanto a los procesos creativos, por lo que se deja un material que puede ser utilizado por cualquier docente de básica secundaria. Asimismo, tiene un valor actitudinal, ya que el estudiante desarrolla un papel activo en su educación musical y desarrolla autonomía.

\section{REFERENCIAS}

Bergee, Martin J. 2003. "Faculty Interjudge Reliability of Music Performance Evaluation". Journal of Research in Music Education 51 (2): 137-150.

Chacón Solís, Liliana Alicia. 2012. “¿Qué significa 'evaluar' en música?". Revista Electrónica Complutense de Investigación en Educación Musical 9: 1-25.

Chow, Jonathan, Haoyang Feng, Robert Amor y Burkhard C. Wünsche. 2013. “Music education using augmented reality with a head mounted display". Proceedings of the Fourteenth Australasian User Interface Conference 139: 73-79.

Collins, Anita. 2014. "Music Education and the Brain What Does It Take to Make a Change?". Update: Applications of Research in Music Education 32 (2): 4-10.

Colwell, Richard. 2002. "Assessment's Potential in Music Education." En The New Handbook of Research on Music Teaching and Learning, editado por Richard Colwell y Carol Richardson, 1128-1158. Nueva York: Oxford University Press. 
Delgado, Roberto Gallego, Nerea Saura Parra y Pedro Miguel Núñez Trujillo. 2013. "AR-Learning: libro interactivo basado en realidad aumentada con aplicación a la enseñanza". Tejuelo: Didáctica de la Lengua y la Literatura. Educación 8: 74-88.

Duke, Robert A. 2010. Intelligent Music Teaching: Essays on the Core Principles of Effective Instruction. Texas: Learning and Behavioural Resources.

Hernández Sampieri, Roberto. 1998. Metodología de la investigación. México: McGraw-Hill.

Navarro, Isidoro. 2014. "Realidad aumentada y educación". educ@conTIC, acceso 16 de septiembre de 2015, http://www.chicagomanualofstyle.org/tools_citationguide.html

Ortegón, Felipe. 2014. “Investigación revela completa radiografía del uso de internet de los adolescentes en Bogotá". Campus 2.0: Noticias, Eventos y Opinión, acceso el 5 de marzo de 2015, En blog Campus 2.0: Noticias, Eventos y Opinión, http://www.unisabana.edu.co/ nc/la-sabana/campus-20/noticia/articulo/investigacion-revela-completa-radiografia-del-uso-deinternet-de-los-adolescentes-en-bogota/

Peula, J. M., J. A. Zumaquero, C. Urdiales, A. M. Barbancho y F. Sandoval. 2016. "Realidad aumentada aplicada a herramientas didácticas musicales." Nuevas Tecnologías Aplicadas a la Educación, acceso el 22 de febrero de 2016, http://cfeliz001.blogspot.com.co/2016/02/ realidad-aumentada-aplicada.html

Ronderos, M. E. y Mantilla, M.T. Ministerio de Educación Nacional. 2015. “Educación artística: serie lineamientos curriculares", acceso el 20 de marzo de 2015, http://www.mineducacion. gov.co/1621/articles-89869_archivo_pdf2.pdf

Raymaekers, Linsey. 2013. "Learning Piano Through an Augmented Piano System". Tesis de grado, Universidad de Hasselt, Bélgica.

Saunders, T. Clark y John M. Holahan. 1997. "Criteria-Specific Rating Scales in the Evaluation of High School Instrumental Performance." Journal of Research in Music Education 45 (2): 259-272.

South Carolina Band Directors Association. 2015. "SC Band Web Site", acceso el 20 de marzo de 2015, http://bandlink.org

Verona, Fran y Javier del Pino. 2015. "Realidad aumentada online de forma fácil - ARcrowd". ARcrowd, acceso el 15 de marzo de 2015, http://arcrowd.com/

Weing, Matthias, Amrei Röhlig, Katja Rogers, Jan Gugenheimer, Florian Schaub, Bastian Könings, Enrico Rukzio y Michael Weber. 2013. "P.I.A.N.O.: enhancing instrument learning via interactive projected augmentation". Proceedings of the 2013 ACM conference on Pervasive and ubiquitous computing adjunct publication 75-78.

Williams, Pharrell. 2013. Happy. Circle House Studios, CD-ROM.

\section{Cómo citar este artículo:}

Amaya Cote, Liliana \& Santoyo Diaz, Julian. 2017. “Evaluación del uso de la realidad aumentada en la educación musical" Cuadernos de Música, Artes Visuales y Artes Escénicas 12 (1): 143-157. doi:10.11144/Javeriana.mavae12-1.urae. 